\title{
Inventario florístico de plantas vasculares en fragmentos de bosque seco tropical en el departamento Magdalena, Colombia
}

\author{
Floristic inventory of vascular plants in fragments \\ of tropical dry forest in the department Magdalena, \\ Colombia
} Acta Botanica Mexicana

\author{
Fran Herazo Vitola1,5 (iD, Dairo Carrascal Prascal (iD, Maziel Herrera Castillo² (iD, Dayana Sofía Valencia-Cuéllar,4 (iD)
}

\section{Resumen:}

Antecedentes y Objetivos: El bosque seco tropical es uno de los ecosistemas más amenazados en el mundo, principalmente por afectaciones antrópicas y ambientales. Se presenta el inventario de la flora vascular en fragmentos de bosque seco durante un periodo de baja precipitación en la subregión Norte, Centro y Sur del departamento Magdalena, Colombia.

Métodos: Se realizó un inventario florístico en seis puntos de muestreo con cinco transectos ( $0.1 \mathrm{ha}$ ) en cada uno, distribuidos de forma aleatoria, sumando un total de 0.6 ha. Se censaron todas las plantas con diámetro a la altura del pecho (DAP) $\geq 1 \mathrm{~cm}$ y altura mínima de $1.5 \mathrm{~m}$ y se registró el hábito de crecimiento. El trabajo de campo fue de noviembre 2019 a febrero de 2020. Las especies se identificaron utilizando claves taxonómicas y consultando colecciones en línea, los nombres se corroboraron en IPNI y el Catálogo de plantas y líquenes de Colombia. Se comprobó la veracidad del muestreo con curvas de acumulación y se efectuó una correlación entre los valores de riqueza y precipitación.

Resultados clave: Se registraron 191 especies, distribuidas en 52 familias, siendo las más representadas Fabaceae (47) y Bignoniaceae (16). La especie más frecuente fue Handroanthus chrysanthus y el hábito de crecimiento fue el de árbol (65\%). El indicador no paramétrico Bootstrap fue el que mejor se ajustó al número de especies esperadas, arrojando un porcentaje de completitud de $87.26 \%$. Los datos de precipitación no se correlacionaron significativamente $(\mathrm{R}=0.53$ y $\mathrm{P}=0.27)$ con los totales de riqueza inventariados.

Conclusiones: Este inventario permitirá generar nuevos aportes científicos de la flora en el bosque seco tropical del departamento Magdalena. La dominancia de especies como Handroanthus chrysanthus y Bursera simaruba puede deberse a las adaptaciones morfológicas expresadas en mecanismos fisiológicos para tolerar periodos sin precipitaciones.

Palabras clave: curvas de acumulación, hábitos, lista de especies, precipitación, riqueza.

\section{Abstract:}

Background and Aims: The tropical dry forest is one of the most threatened ecosystems in the world, mainly due to anthropic and environmental effects. The inventory of vascular flora in dry forest fragments during a period of low rainfall in the subregion North, Center and South of the department Magdalena, Colombia, is presented.

Methods: A floristic inventory was carried out, at six sampling points with five transects ( $0.1 \mathrm{ha}$ ) in each one, distributed randomly, totaling 0.6 ha. All plants with a diameter at breast height $(\mathrm{DBH}) \geq 1 \mathrm{~cm}$ and a minimum height of $1.5 \mathrm{~m}$ were included in the census and the growth form was recorded. Fieldwork was carried out from November 2019 to February 2020. The species were identified using taxonomic keys and consulting online collections, the names were corroborated in IPNI and the Catalogue of plants and lichens of Colombia. The veracity of the sampling was verified through accumulation curves and a correlation was made between the values of richness and precipitation.

Key results: One hundred ninety-one species were registered, distributed in 52 families, the most represented being Fabaceae (47) and Bignoniaceae (16). The most frequent species was Handroanthus chrysanthus and the growth habit was the tree (65\%). The non-parametric Bootstrap indicator was the most suitable for the number of expected species, providing a completeness percentage of $87.26 \%$. The precipitation data were not correlated significantly $(\mathrm{R}=0.53$ y $\mathrm{P}=0.27$ ) with the total richness inventoried.

Conclusions: This inventory will allow generating new scientific contributions of the flora in the tropical dry forest of the Magdalena department. The dominance of species such as Handroanthus chrysanthus and Bursera simaruba may be due to morphological adaptations expressed in physiological mechanisms to tolerate periods without precipitation.

Key words: accumulation curves, climatic variability, habits, precipitation, richness, species list.

${ }^{1}$ Universidad de Sucre, Carrera 28 No. 5-267, Sincelejo, Sucre, Colombia.

${ }^{2}$ Fundación Universitaria Tecnológico Comfenalco, Cr. 44 \#30a-91, Cartagena, Bolívar, Colombia.

${ }^{3}$ Universidad de la Costa CUC, Calle 58 \#55-66, BarranquiIla, Colombia.

${ }^{4}$ Universidad del Magdalena, Facultad de Ciencias Básicas, Grupo de Investigación en Insectos Neotropicales, Carrera 32 \#22-08, 470004 Santa Marta, Colombia.

${ }^{5}$ Autor para la correspondencia: fran.herazo@unisucre. edu.co
Recibido: 9 de diciembre de 2020.

Revisado: 16 de marzo de 2021

Aceptado por Marie-Stéphanie Samain: 10 de junio de 2021.

Publicado Primero en línea: 13 de julio de 2021

Publicado: Acta Botanica Mexicana 128 (2021).
Citar como: Herazo Vitola, F., D. Carrascal Prasca, M. Herrera Castillo y D. S. Valencia-Cuéllar. 2021. Inventario florístico de plantas vasculares en fragmentos de bosque seco tropical en el departamento Magdalena, Colombia. Acta Botanica Mexicana 128: e1828. DOI: https://doi. org/10.21829/abm128.2021.1828 


\section{Introducción}

Los bosques secos tropicales (BST) en Colombia son una de las áreas más amenazadas y afectadas de la progresiva degradación en las últimas décadas; se registra menos de $10 \%$ de su cobertura original, producto de actividades como la ganadería extensiva, agricultura, minería y deforestación (Klein et al., 2014; Pizano et al., 2014). Un factor preponderante como la variabilidad climática también ha estado incidiendo históricamente en sus procesos funcionales (regeneración, simplificación en la estructura y composición) (Noguera, 2016). Según Choat et al. (2012), todos los biomas forestales están expuestos a fenómenos climáticos, determinados por extensos periodos de sequía. En efecto, las variaciones locales del clima, expresadas por gradientes ambientales como la temperatura, precipitación y humedad, han modificado la distribución actual del bosque (Pabón y Montealegre, 2000).

En Colombia, el BST se encuentra distribuido en varias regiones del país, abarcando las del Caribe, Norandina (sectores de Santander y Norte de Santander), los valles Interandinos de los ríos Cauca y Magdalena, El Patía (Nariño y Cauca) y Los Llanos en los departamentos Arauca, Casanare, Meta y Vichada (IAVH, 1998; Galvis y Mesa, 2014). Esta distribución podría cambiar a futuro por fenómenos climáticos y antrópicos. Por ejemplo, Alvarado y Ospina (2015) encontraron que aproximadamente $80 \%$ de la cobertura en el Valle del Cauca se localiza en gran medida en los ecosistemas de montaña, en zonas transicionales de bosque seco a húmedo, cuyo comportamiento podría estar mostrando procesos adaptativos frente a efectos antrópicos excesivos. Las posibles consecuencias de las variaciones ambientales sobre la vegetación han sido analizadas en algunas ocasiones. Un caso particular es el estudio de Alarcón y Pabón (2013), quienes detectaron el aumento y disminución de algunos ecosistemas por cambios del clima (Wright, 2005).

El BST en el departamento Magdalena presenta una extensión aproximada de 94.625 ha, de las cuales $39.8 \%$ se encuentran en el municipio Aracataca, $0.8 \%$ en el municipio Sabanas de San Ángel, $8.48 \%$ en Plato, y $1.97 \%$ en Santa Bárbara de Pinto, según la cartografía del Instituto Alexander Von Humboldt (IAVH, 2014). Es decir, se trata de un área reducida que abarca este ecosistema en tierras bajas del departamento, las cuales han sido poco o nulamente analizadas, ya que la mayoría de los esfuerzos se han concentrado en tierras altas de la Sierra Nevada de Santa Marta (Rangel y Garzón, 1995; Dechner y Diazgranados, 2007; Pinto y Rangel, 2010), o en lugares con alguna categoría de protección (Carbonó y García, 2010; García et al., 2021). Ante este panorama, se promueven estrategias como el proyecto de investigación "Efectos de la variabilidad climática y el cambio climático sobre el recurso hídrico, biodiversidad y actividades agropecuarias en el departamento del Magdalena", que busca generar conocimientos que permitan explicar la situación ambiental actual del departamento. Teniendo en cuenta lo anterior, en el presente artículo se abordan los resultados de un inventario florístico de las plantas vasculares en fragmentos de BST en un periodo de baja precipitación en la subregión Norte, Centro y Sur del departamento Magdalena, Colombia.

\section{Materiales y Métodos}

\section{Área de estudio}

El área de interés se ubica en el departamento Magdalena, situado al norte de Colombia, tiene una extensión de $23.188 \mathrm{~km}^{2}$ que representan aproximadamente $2 \%$ del territorio nacional y $15 \%$ del área del Caribe, el cual incluye ríos, ciénagas y embalses (CORPAMAG y UA, 2015). Según el diagrama de Holdridge (1967), la zona de vida presente es BST propio de tierras bajas, el cual ocupa un ámbito enmarcado por promedios de temperatura superiores a $24{ }^{\circ} \mathrm{C}$. Se presenta en el piso térmico cálido, en altitudes desde el nivel del mar hasta $1000 \mathrm{~m}$. Las relaciones de evapotranspiración potencial son entre 0.8 y 2, con precipitación total anual entre 800 y 2000 mm (García et al., 2012). Las épocas climáticas secas se encuentran enmarcadas entre diciembre y febrero y la húmeda de septiembre a noviembre en relación con los vientos alisios (Ruiz y Bernal, 2009).

EI BST en el departamento se concentra sobre las estribaciones de la Sierra Nevada de Santa Marta (subregión Norte).

Para este estudio se trabajó en fragmentos de la parte baja del municipio Aracataca; asimismo, a nivel de los municipios Sabanas de San Ángel, Plato (subregión centro) y Santa Bárbara de Pinto (subregión sur) sobre relieves llanos cerca el río Magdalena (CORPAMAG y UA, 2015) (Fig. $1)$. 
En el municipio Aracataca, se laboró en tres fincas: La Patagonia $\left(10^{\circ} 37^{\prime} 24.5290^{\prime \prime} \mathrm{N}, 74^{\circ} 03^{\prime} 30.9348^{\prime \prime} \mathrm{W}\right)$, Monatos $\left(10^{\circ} 32^{\prime} 29.6376^{\prime \prime} \mathrm{N}, 74^{\circ} 04^{\prime} 16.2372^{\prime \prime} \mathrm{W}\right)$ y Villa Lorena $\left(10^{\circ} 31^{\prime} 18.4800^{\prime \prime} \mathrm{N}, 74^{\circ} 04^{\prime} 36.6888^{\prime \prime} \mathrm{W}\right)$; la vegetación en esta área se encuentra sobre un paisaje de lomeríos con relieves en filas y vigas con llanuras aluviales determinadas por los ríos Tucurinca, Aracataca y Fundación (IDEAM et al., 2007). En el municipio Sabanas de San Ángel, se traba- jó en la finca Italia $\left(10^{\circ} 01^{\prime} 36.1272^{\prime \prime} \mathrm{N}, 74^{\circ} 12^{\prime} 29.7792^{\prime \prime W}\right)$, en el municipio Plato, en la finca Tacaloa $\left(9^{\circ} 46^{\prime} 49.962^{\prime \prime} \mathrm{N}\right.$, $74^{\circ} 34^{\prime} 55.1172$ 'W) y en el municipio Santa Bárbara de Pinto en la finca Silvaderal $\left(9^{\circ} 36^{\prime} 0.504^{\prime \prime} \mathrm{N}, 74^{\circ} 38^{\prime} 53.7972^{\prime \prime} \mathrm{W}\right)$ (Fig. 1). Estos puntos se encuentran en parches aislados de bosque en paisajes de montañas y lomeríos, influenciados por la cuenca del río Magdalena, con relieve de ondulaciones leves (IDEAM et al., 2007).

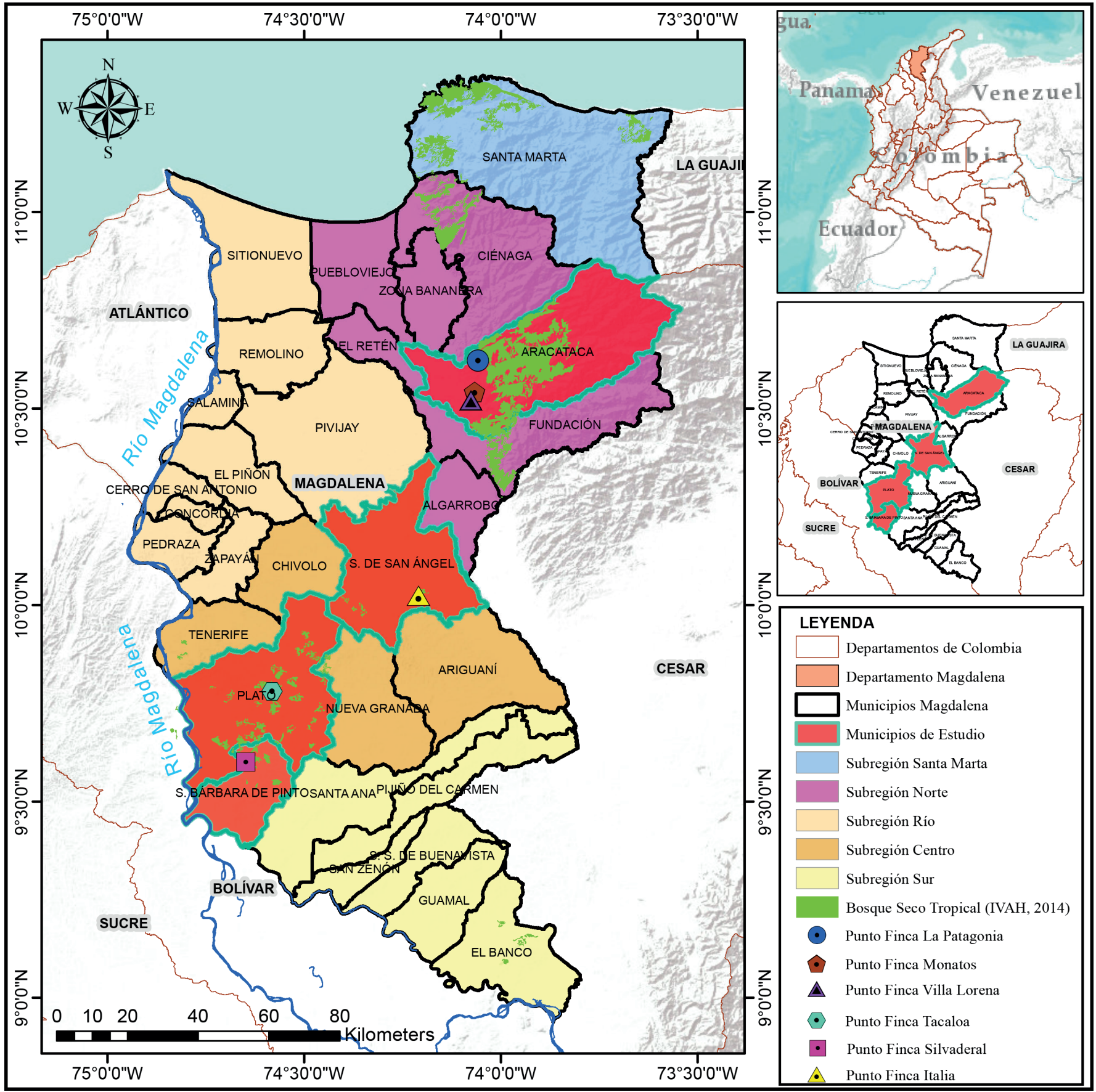

Figura 1: Distribución de puntos de muestreo en fragmentos de bosque seco tropical del departamento Magdalena, Colombia. 


\section{Metodología de inventarios}

En cada finca se levantaron cinco transectos, para un área de 0.1 ha, distribuidos de forma aleatoria en los fragmentos de BST, totalizando 0.6 ha en 30 transectos, utilizando el método propuesto por Gentry (1982) con modificaciones de Álvarez et al. (2001) y Mendoza (1999). El censo incluyó todas las plantas con diámetro a la altura del pecho (DAP) $\geq 1 \mathrm{~cm}$ y altura mínima de $1.5 \mathrm{~m}$, la cual se estimó y registró en campo para cada individuo, incluyendo el hábito de crecimiento.

Los municipios Santa Bárbara de Pinto y Plato se visitaron en noviembre de 2019, cuando aún se registraban algunas Iluvias (150 y 210 mm mensuales) (Fig. 2), marcando el inicio de la época seca, la cual se evidenció por completo en febrero de 2020 cuando se muestreó en el municipio Aracataca, demostrada por bajos valores pluviométricos ( 0 y $4 \mathrm{~mm}$ ) (Fig. 3).

Los datos de precipitación registrados por las estaciones climatológicas locales se obtuvieron del Instituto de Hidrología, Meteorología y Estudios Ambientales (IDEAM, 2020).

Las especies se identificaron mediante claves taxonómicas (Smith y Fernández, 1954; Gentry, 1996; Mendoza et al., 2004; Forero y Romero, 2005; Gentry, 2009; Morales, 2009; Rangel, 2012). Además, se compararon con ejemplares de colecciones en línea, tales como la del Herbario de la Universidad Nacional (UNAL, 2019), Jardín Botánico de Missouri (TROPICOS, 2020), United States Botanical Garden (USBG, 2020), JSTOR Global Plants (JSTOR, 2020) y el Catálogo de Plantas y Líquenes de Colombia (Bernal et al., 2019). Los nombres científicos de cada especie fueron corroborados en el International Plant Name Index (IPNI, 2020) y el Catálogo de Plantas y Líquenes de Colombia (Bernal et al., 2019).

\section{Análisis}

Se verificó el esfuerzo de muestreo (completitud) mediante curva de acumulación con estimadores no paramétricos basados en las especies raras (Chao 2, Jacknife 1, ICE, Jacknife 2 y Bootstrap), de los cuales se abordó el que mejor se ajustó a los datos obtenidos para todos los puntos. Dichos análisis se realizaron con el software EstimateS v. 9.10 (Colwell, 2013), según lo descrito por Jiménez y Hortal (2003). Adicionalmente, los datos de precipitación del IDEAM (2020) se interpolaron para generar mapas de isoyetas de precipitación mensuales empleando el programa ArcGIS v.

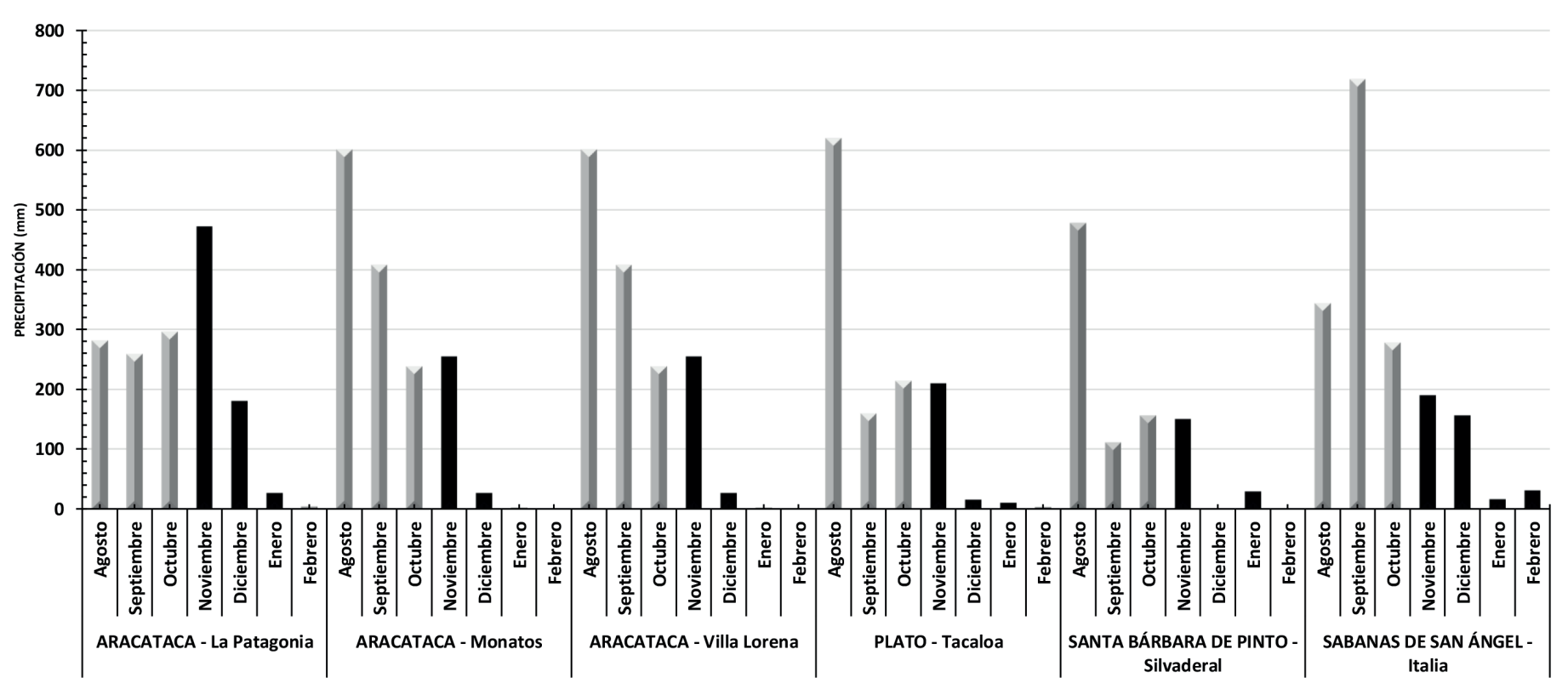

Figura 2: Precipitación mensual durante el muestreo en fragmentos de bosque seco tropical por puntos de muestreo y municipios de interés del departamento Magdalena, Colombia. 

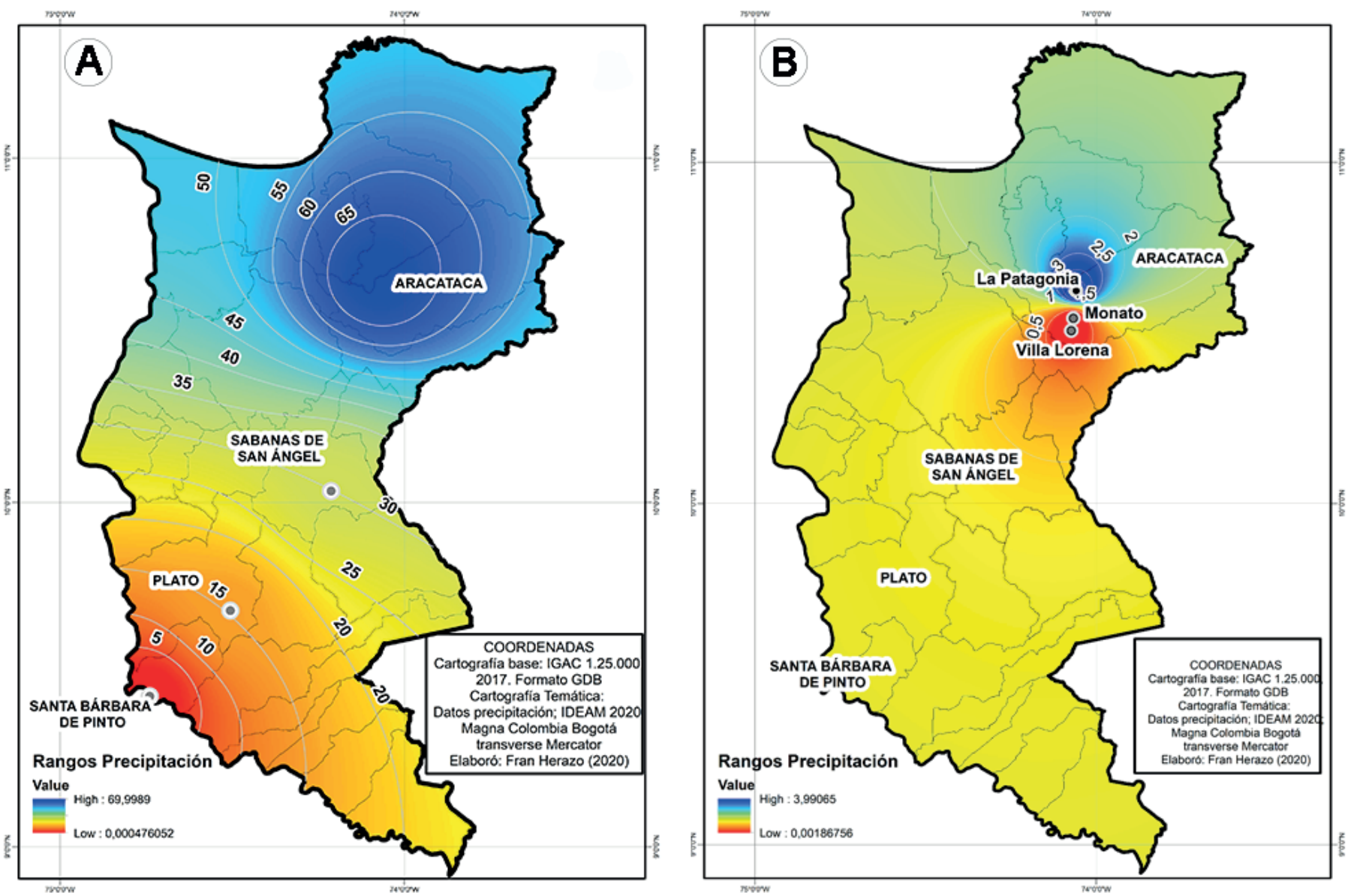

Figura 3: Mapas interpolados de precipitación para los meses de noviembre 2019 (A) y febrero 2020 (B), durante el muestreo sobre fragmentos de bosque seco tropical, en los municipios Sabanas de San Ángel, Plato, Santa Bárbara de Pinto y Aracataca, del departamento Magdalena, Colombia.

10.3 (ESRI, 2015). Con estos valores y los de riqueza, se realizó una correlación simple para identificar cualquier tipo de correspondencia mediante el programa STATISTICA v. 6 (Statsoft, 2001).

\section{Resultados}

\section{Inventario florístico}

En 0.6 ha de fragmentos de BST de los municipios priorizados para el departamento Magdalena, se registró un total de 191 especies (Fig. 4), entre árboles, arbustos, lianas y palmas distribuidas en 52 familias. Fabaceae (47 especies) y Bignoniaceae (16 especies) fueron las más numerosas. En general, Handroanthus chrysanthus (Jacq.) S.O. Grose fue la especie más abundante (Apéndice). En cuanto al hábito de crecimiento, el más representado fue el de árbol (65\%), seguido por liana (19\%), arbusto (14\%), y palma (2\%) (Fig. 5).
La distribución del número de especies se concentró en los municipios Santa Bárbara de Pinto, finca Silvaderal (68) y Plato, finca Tacaloa (67) con los mayores valores. En contraste, Aracataca, finca Monatos (51) y Aracataca, La Patagonia (55) reportaron los menores números (Apéndice). El mayor porcentaje de especies se evidenció durante el mes de noviembre en los municipios Santa Bárbara de Pinto, finca Silvaderal y Plato, finca Tacaloa (19\% cada uno), mientras que los menores porcentajes se presentaron durante el mes de febrero, en el municipio Aracataca, finca Monatos (14\%) y Aracataca, finca La Patagonia (15\%).

\section{Esfuerzo de muestreo}

De acuerdo con el total de especies obtenido, el indicador no paramétrico Bootstrap fue el que mejor se ajustó al número de especies esperadas, arrojando el mayor porcenta- 

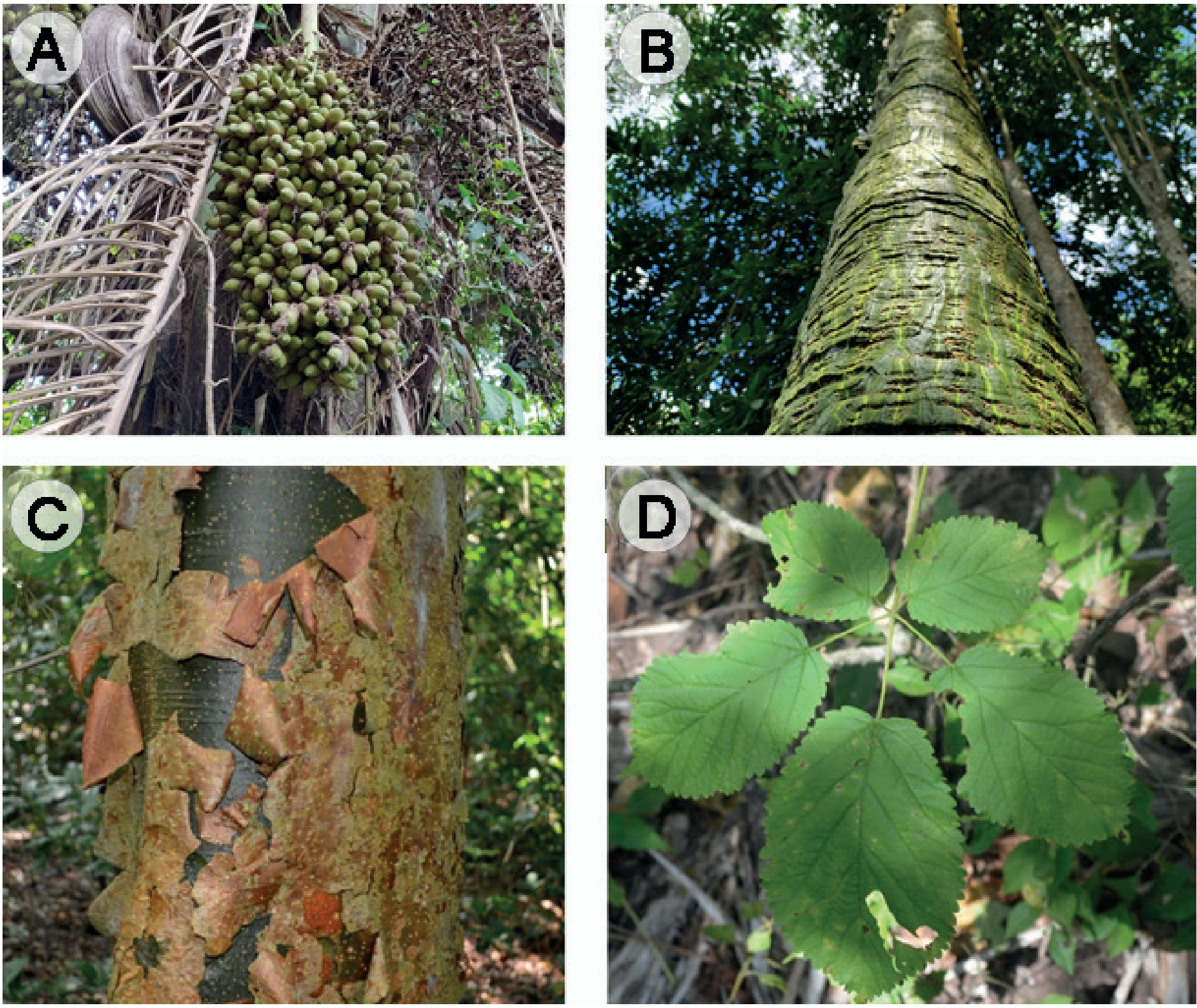

Figura 4: Especies del bosque seco tropical, departamento Magdalena, Colombia. A. Attalea butyracea (L.f.) Wess. Boer; B. Pseudobombax septenatum (Jacq.) Dugand; C. Bursera simaruba (L.) Sarg.; D. Handroanthus chrysanthus (Jacq.) S.O. Grose. Fotografías: A y B: F. Herazo Vitola; C y D: D. Carrascal Prasca.

je de completitud $87.26 \%$, con respecto a ICE (77.64), Chao 2 (79.98), Jack 1 (76.41) y Jack 2 (69.59) (Fig. 6).

Según Bootstrap, los fragmentos de BST ubicados en los municipios Santa Bárbara de Pinto, finca Silvaderal (88.50 \%) y Aracataca, finca Monatos (87.53\%), alcanzaron los mayores porcentajes de especies registradas, mientras que en el municipio Aracataca, finca La Patagonia (85.21\%) se obtuvo el menor porcentaje (Fig. 7).

\section{Discusión}

\section{Inventario florístico}

El listado de especies entre los seis fragmentos, ubicados en cuatro municipios estudiados en el departamento Magdalena, permite conocer el estado actual del BST en un periodo de baja precipitación, siendo los resultados obtenidos con DAP $\geq 2.5 \mathrm{~cm}$ bajos en comparación con otras localidades del Caribe colombiano con el mismo diámetro en 0.1 ha, no obstante, las fincas Tacaloa (62 especies) en el mu- 


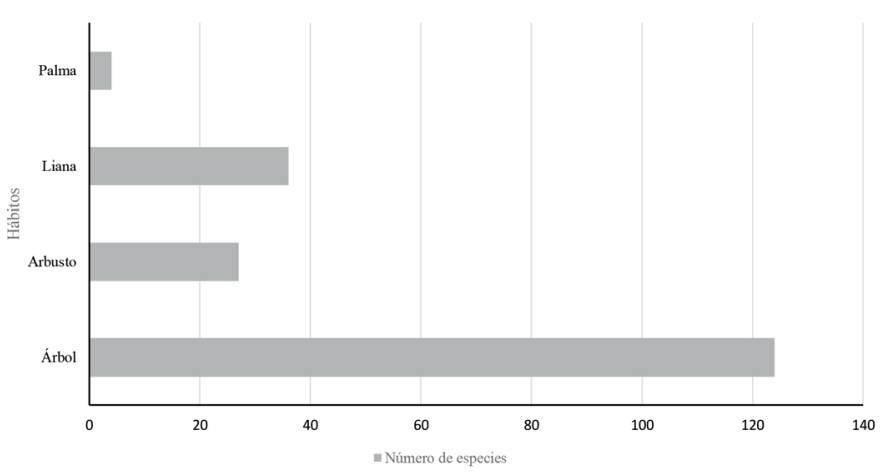

Figura 5: Número de especies por hábitos de crecimientos reportadas durante el muestreo en fragmentos de bosque seco tropical en los municipios Aracataca, Sabanas de San Ángel, Santa Bárbara de Pinto y Plato del departamento Magdalena, Colombia.

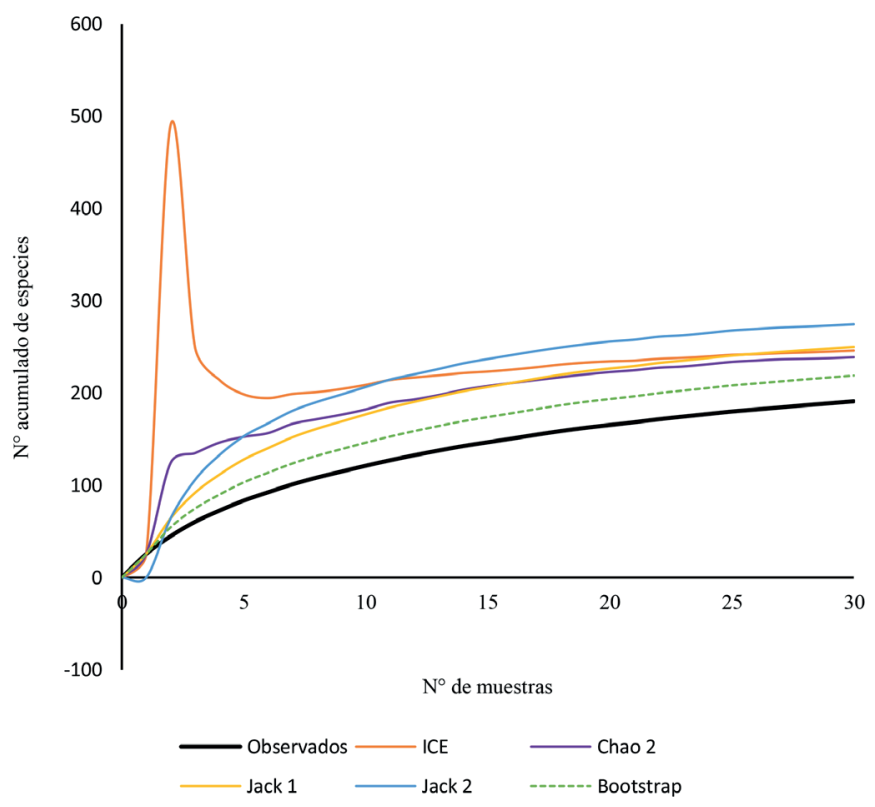

Figura 6: Curvas de acumulación de especies para definir la completitud de los muestreos en fragmentos de bosque seco tropical en los municipios Aracataca, Sabanas de San Ángel, Santa Bárbara de Pinto y Plato del departamento Magdalena, Colombia.

nicipio Plato, y Villa Lorena (59 en el municipio Aracataca, superaron lo reportado en los municipios Galerazamba (55) y Tierra Bomba (56) en el departamento Bolívar (Mendoza, 1999). Al contrastar con datos reportados con DAP $\geq 1 \mathrm{~cm}$ en un estudio en los Montes de María en el departamento Sucre en 0.1 ha (Paraíso con 120 (municipio Colosó), Pajarito con 110 (municipio Chalán), Garrapata con 104 (muni-

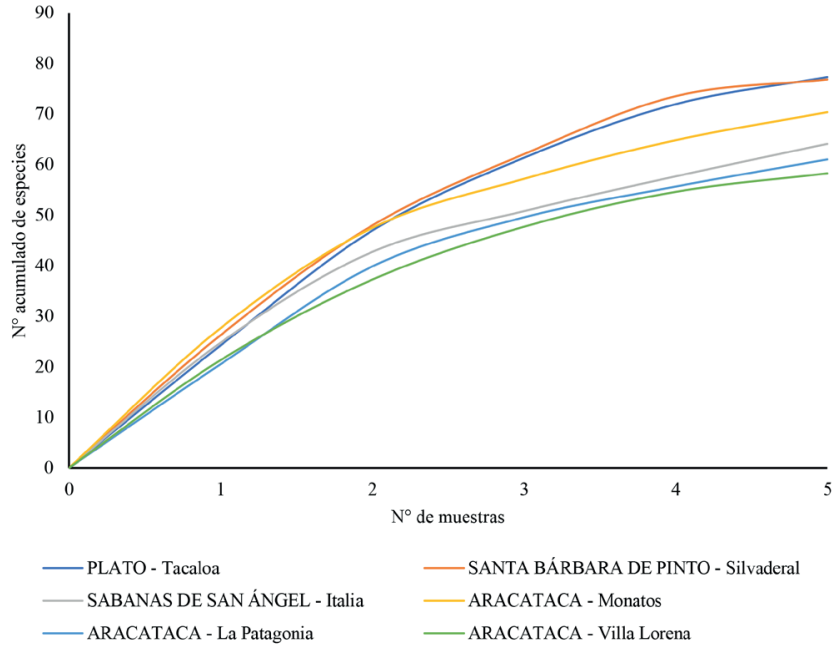

Figura 7: Curvas de acumulación de especies, durante el muestreo en fragmentos de bosque seco tropical, del indicador Bootstrap para los municipios Aracataca, Sabanas de San Ángel, Santa Bárbara de Pinto y Plato del departamento Magdalena, Colombia.

cipio San Onofre), El Cacao con 111 (municipio San Onofre) y Roca Madre con 122 (municipio Toluviejo), Herazo et al., 2017), realizado en distintas épocas, sus resultados superaron lo encontrado en este estudio, posiblemente por el cubrimiento estacional.

Los municipios Plato y Santa Bárbara de Pinto obtuvieron los mayores números de especies, lo que concuerda con su amplia cobertura vegetal y poca perturbación en comparación con el resto de las zonas. Lo anterior también puede explicarse por la ausencia de algunas especies, como Attalea butyracea (L.f.) Wess. Boer, reportada frecuentemente en Sabanas de San Ángel, siendo su crecimiento poblacional influenciado por la actividad ganadera, ya que sus semillas son dispersadas por el ganado, indicando zonas con áreas intervenidas (CAR, 2015).

La familia Fabaceae fue la más dominante en el estudio; la cual es uno de los grupos con mayor diversidad y amplia distribución en los trópicos (Gentry, 1995). Sus características morfológicas reportadas, tales como hojas compuestas, presencia de espinas y tricomas, entre otras (Gentry, 1996), disminuyen la exposición a la radiación solar y les permiten soportar la sequía (Ceroni, 2003). Algunos integrantes también tienen la capacidad de regular la concentración de nitrógeno en el suelo (Herrera et al., 2017). Este es un aspecto importante, ya que la intensificación de 
los ciclos hidrológicos (sequía-lluvia) están relacionados con las variaciones de sus concentraciones (Morillas et al., 2015), lo cual puede sugerir que estas especies podrían ser utilizadas en procesos de restauración frente a escenarios drásticos de cambio climático (Herrera et al., 2017).

En cuanto a las especies más abundantes, Handroanthus chrysanthus fue la que presentó el mayor número de individuos, siendo observada en estado de floración durante el muestreo. Su amplia distribución sobre los extensos paisajes de lomeríos en la cuenca baja del río Magdalena puede deberse a la capacidad de soportar periodos de sequía y dispersar semillas durante ese tiempo, ya que, ante la deshidratación, pueden almacenar recursos de agua en sus tallos, liberar sus hojas y generar flores (Borchert, 1994). Este fenómeno morfo-fisiológico consiste en la desactivación de los meristemos foliares, lo que estimula la activación de los primordios florales (Reich y Borchert, 1982).

Por su parte, el hábito de crecimiento de árbol fue el más representado entre los municipios de estudio, seguido por lianas, arbustos y palmas. El dominio del hábito arbóreo es un patrón reportado en muchos trabajos realizados en otros fragmentos de BST del país, según lo exponen Vargas (2012) y Londoño y Torres (2015). Sin embargo, el de lianas también sobresalió, el cual es poco dominante en el interior de estos ecosistemas, ya que, generalmente, colonizan el borde de los bosques y las áreas abiertas (Van Der Heijden y Phillips, 2009), aunque algunos trabajos, como el de Villanueva et al. (2015), asocian este patrón con bosques maduros. No obstante, el predominio de esta forma de vida en el área de estudio sugiere bosques influenciados por periodos secos prolongados. Por tanto, este fenómeno podría modificar la dinámica estructural de estos bosques (Arellano, 2015).

\section{Esfuerzo de muestreo}

Villarreal et al. (2006) indican que a partir de $85 \%$ de registros el muestreo es completo, concordando con los resultados presentados como representativos. Cabe resaltar que el indicador Bootstrap es muy robusto al momento de estimar la variación partiendo de datos de presencia y ausencia y es fiable al realizar un inventario regional (Méndez et al., 2005; Cristóbal et al., 2014).

\section{Precipitación}

Los datos de precipitación no se correlacionaron significativamente $(R=0,53$ y $P=0.27)$ con la riqueza inventariada (Fig. 8). Sin embargo, en áreas como Plato y Santa Bárbara de Pinto, donde la pluviosidad fue superior (0-69 $\mathrm{mm}$ ) durante el mes de noviembre, la riqueza fue alta, en comparación con Aracataca en el mes de febrero, en plena época de sequía, donde la precipitación fue nula o muy baja (0-4 mm mensual).

Cabe mencionar que en el municipio Aracataca, finca Villa Lorena, a pesar de no registrar precipitación, mostró una alta riqueza en relación con Sabanas de San Ángel, donde la pluviosidad fue superior y la riqueza fue baja, posiblemente sugiriendo a la finca Villa Lorena (Aracataca) como un sector con elementos biológicos fotosintéticos con procesos adaptativos ante fenómenos de variabilidad climática. Tal es el caso de Bursera simaruba (L.) Sarg., especie observada en dos fases fenológicas (floración y fructificación) durante el periodo seco, la cual puede ser considerada potencialmente como indicadora de estas variaciones locales, debido a sus características fenológicas como respuesta a las condiciones ambientales (Basilio, 2017). También es relevante el caso de Pseudobombax septenatum (Jacq.) Dugand, la cual se encontró en las tres fincas en el municipio Aracataca (La Patagonia, Monatos y Villa Lorena) en estado de fructificación y distribución de semillas. Según Berdugo y Rangel (2015), las poblaciones de esta especie tienden a sincronizarse durante las fases fenológicas reproductivas y periodos de poca precipitación.

En términos de fragilidad, los fragmentos de BST en las fincas La Patagonia y Monatos (ambas en Aracataca) podrían verse afectados ante el comportamiento climático local. De acuerdo con Kohler et al. (2014), los eventos de variabilidad climática, como los de sequía prolongada, perturban mayormente los bosques ubicados en paisajes de montañas, que a los de tierras bajas.

\section{Conclusiones}

A pesar del alto nivel de fraccionamiento del BST, esta investigación muestra que los fragmentos estudiados en el departamento Magdalena aún conservan una diversidad importante y que como estrategia a la sequía tienden a aumentar los individuos lianescentes de porte bajo. En térmi- 


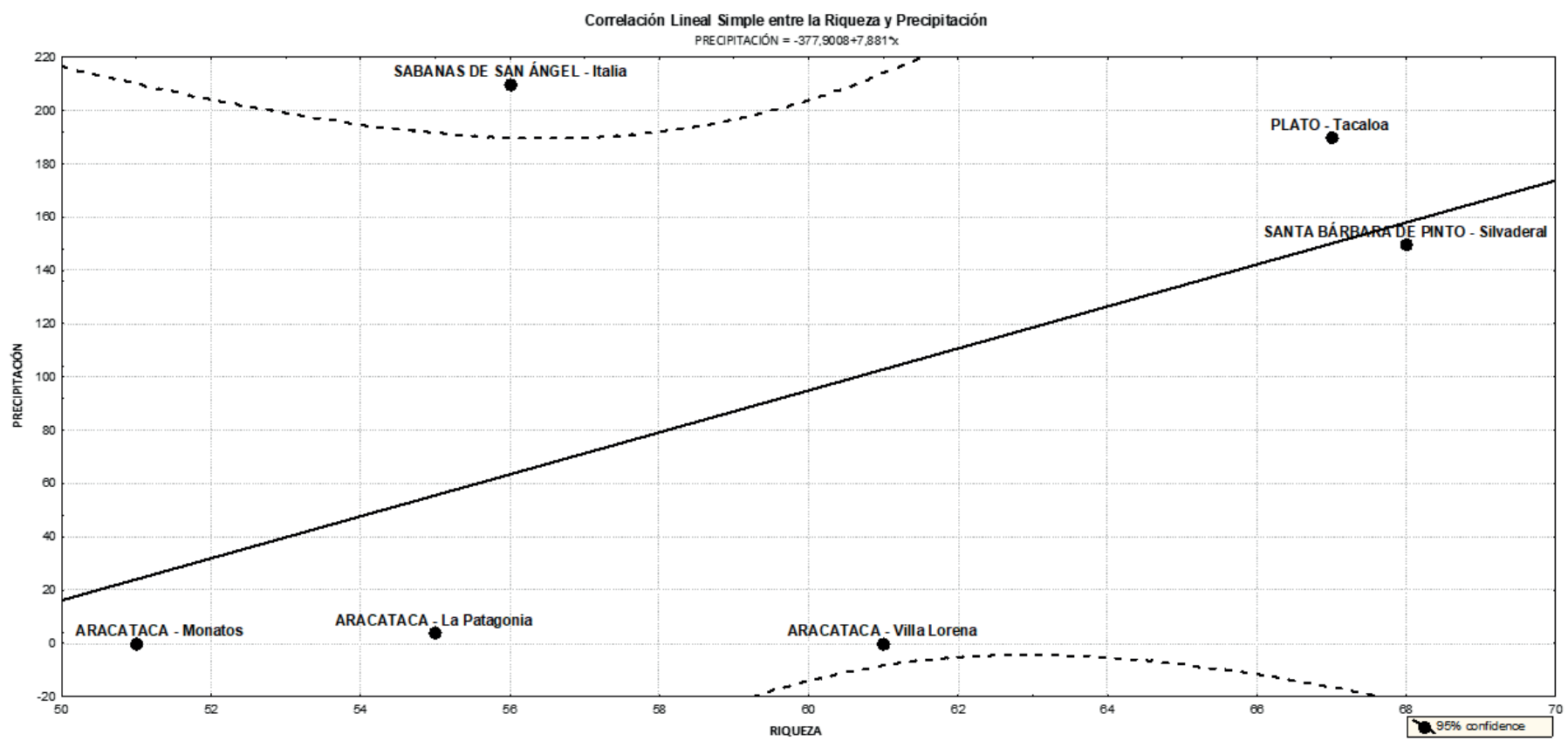

Figura 8: Correlación lineal simple entre la riqueza y precipitación reportada durante el muestreo en fragmentos de bosque seco tropical para los municipios Aracataca, Sabanas de San Ángel, Santa Bárbara de Pinto y Plato del departamento Magdalena, Colombia.

nos de adaptabilidad a los periodos de poca precipitación, los municipios Santa Bárbara de Pinto y Plato parecen soportar mejor este tipo de alteraciones locales, ya que reportaron los mayores valores de registros de especies. Así mismo, fue sobre estos donde se observó menor intervención antrópica, lo cual también puede explicar el hallazgo de estos resultados.

La riqueza reportada se torna en generación de nuevos conocimientos y aportes científicos de la flora en periodos de baja precipitación para el BST del departamento, siendo los estudios y registros pocos o nulos en los municipios muestreados. A pesar de no encontrar diferencias significativas entre la correlación de la variable precipitación y la riqueza, se observó en algunos puntos, como los del municipio de Aracataca, cambios florísticos en la ausencia de especies de porte bajo y floración de árboles durante el mes con menor pluviosidad (febrero).

La familia con mayor número de especies fue Fabaceae, posiblemente por la plasticidad y adaptabilidad que pueden expresar los miembros que la conforman, concordando con estudios realizados para BST en Colombia (Men- doza, 1999; Albesiano y Fernández, 2006; Carbonó y García, 2010; Torres et al., 2012; Vargas et al., 2016; Herazo et al., 2017). La dominancia de especies como Handroanthus chrysanthus y Bursera simaruba es tal vez producto de la capacidad morfológica expresada en mecanismos fisiológicos para tolerar periodos sin precipitaciones, como un escenario típico de variabilidad climática. Por otra parte, los inventarios realizados con los tamaños de áreas muestreados indicaron ser representativos para el BST según el estimador Bootstrap, el cual fue el que más se ajustó a lo esperado.

\section{Contribución de autores}

FHV, DCP y DSVC participaron en la fase de campo; FHV y DCP identificaron las especies y realizaron el inventario; FHV y DCP procesaron los datos de campo; FHV analizó los datos y escribió el manuscrito con apoyo de MHC; DCP, DSVC y MHC revisaron el manuscrito; DCP elaboró el cuadro 1 y las figuras 1, 6 y 7; MHC elaboró las figuras 2 y 5; FHV elaboró las figuras 3 y 8 . Todos los autores contribuyeron a la aprobación del manuscrito final. 


\section{Financiamiento}

Este estudio fue financiado por el Sistema General de Regalías Fondo de Ciencia, Tecnología e Innovación del departamento del Magdalena a través del proyecto "Investigación de los efectos de la variabilidad climática y el cambio climático sobre el recurso hídrico, biodiversidad y actividades agropecuarias en el departamento del Magdalena" (Gobernación del Magdalena, Contrato 0728/18), Universidad de La Costa, Barranquilla, Colombia, cambioclimatico@cuc.edu.co.

\section{Agradecimientos}

Al equipo técnico del proyecto mencionado arriba, por su apoyo y acompañamiento en cada parte de esta investigación. A la Universidad de la Costa (CUC), por otorgarnos la oportunidad de ejecutar el estudio y contribuir en la generación de nuevos conocimientos. A la Corporación NaturalSIG, por el suministro de equipos y herramientas técnicas para la recolección de información. A la Universidad de Sucre, por la disposición de espacios de trabajo. A Guiomar Aminta Jáuregui Romero por sus comentarios y sugerencias para mejorar el manuscrito.

\section{Literatura citada}

Alarcón, J. y J. Pabón. 2013. El cambio climático y la distribución espacial de las formaciones vegetales en Colombia. Colombia Forestal 16: 171-185.

Albesiano, S. y J. Fernández. 2006. Catálogo comentado de la flora vascular de la franja tropical (500-1200m) del Cañón del río Chicamocha (Boyacá-Santander, Colombia). Primera parte. Caldasia 28: 23-44.

Alvarado, D. y J. Ospina. 2015. Distribución espacial del bosque seco tropical en el Valle del Cauca, Colombia. Acta Biológica Colombiana 20: 141-153. DOI: https://doi.org/10.15446/ abc.v20n3.46703

Álvarez, E., A. Cogollo, H. Rincón, D. Benítez, T. Parra, W. Rodríguez, A. Idárraga, C. Velásquez y E. Jiménez. 2001. Propuesta metodológica de parcelas normatizadas para los inventarios de vegetación. Convenio Interconexión Eléctrica S.A. y Jardín Botánico Joaquín Antonio Uribe (ISA-JAUM). Documento de trabajo. Versión 1.7. Medellín, Colombia.

Arellano, N. 2015. Evaluación de diversidad taxonómica y funcional de lianas en el gradiente altitudinal Caribe-Villa Mills, Costa Rica. Tesis de maestría. Centro Agronómico Tropical de Investigación y Enseñanza (CATIE). Turrialba, Costa Rica. 69 pp.
Basilio, M. 2017. Línea base de especies vegetales indicadoras adaptadas a la variación climática, para el monitoreo de los bosques de la parte alta y media de la cuenca del Río Nahualate. Gobierno de Guatemala, Ministerio de Ambiente y Recursos Naturales. Guatemala, Guatemala. 120 pp.

Berdugo, M. y J. Rangel. 2015. Composición florística del bosque tropical seco del santuario "Los Besotes" y fenología de especies arbóreas dominantes (Valledupar, Cesar, Colombia). Colombia Forestal 18: 87-103. DOI: https://doi.org/10.14483/ udistrital.jour.colomb.for.2015.1.a05

Bernal, R., S. Gradstein y M. Celis. 2019. Catálogo de plantas y líquenes de Colombia. Instituto de Ciencias Naturales, Universidad Nacional de Colombia. Bogotá, D.C., Colombia. http://catalogoplantasdecolombia.unal.edu.co (consultado marzo de 2020).

Borchert, R. 1994. Soil and stem water storage determine phenology and distribution of tropical dry forest trees. Ecology 75(5): 1437-1449. DOI: https://doi.org/10.2307/1937467

CAR. 2015. Implementación de una acción para la conservación y uso sostenible de la palma Attalea butyracea en la jurisdicción CAR. Corporación Autónoma Regional de Cundinamarca (CAR). Dirección de Modelamiento, Monitoreo y Laboratorio Ambiental. Contrato de Prestación de servicios 1038 de 2015. Bogotá, D.C., Colombia. 38 pp.

Carbonó, E. y H. García. 2010. La vegetación terrestre en la ensenada de Neguanje, Parque Nacional Natural Tayrona (Magdalena, Colombia). Caldasia 32: 235-256.

Ceroni, A. 2003. Distribución de las leguminosas de la parte alta de la cuenca La Gallega. Morropón. Piura. Ecología Aplicada 2(1): 9-13.

Colwell, R. 2013. Statistical estimation of species richness and shared species from samples, Versión 9.10. http://purl.oclc.org/ estimates.

CORPAMAG y UA. 2015. Caracterización, diagnóstico y Análisis de vulnerabilidades y amenazas en el departamento del Magdalena. Diagnóstico Biofísico, Capitulo 6. Corporación Autónoma Regional del Magdalena (CORPAMAG). Santa Marta, Colombia. Pp. 258-308.

Cristóbal, C., J. Méndez, J. Canche y E. Hernández. 2014. Indicadores de diversidad, estructura y riqueza para la conservación de la biodiversidad vegetal en los paisajes rurales. Tropical and Subtropical Agroecosystems 17: 185-196.

Choat, B., S. Jansen, T. Brodribb, H. Cochard, S. Delzon, R. Bhaskar, S. Bucci, T. Feild, S. Gleason, U. Hacke, A. L. Jacobsen, F. Lens, 
H. Maherali, J. Martínez-Vilalta, S. Mayr, M. Mencuccini, P. J. Mitchell, A. Nardini, J. Pittermann, R. B. Pratt, J. S. Sperry, M. Westoby, I. J. Wright y A. E. Zanne. 2012. Global convergence in the vulnerability of forests to drought. Nature 491: 752-755. DOI: https://doi.org/10.1038/nature11688

Dechner, A. y M. Diazgranados. 2007. Composición y estructura de la vegetación boscosa de la cuenca baja del río San Salvador, vertiente norte de la Sierra Nevada de Santa Marta, Colombia. Universitas Scientiarum 12(2): 99-124.

ESRI. 2015. ArcGIS Desktop: Version 10.3. Environmental Systems Research Institute. Redlands, CA., USA. https://enterprise. arcgis.com/en/portal/10.3/use/what-s-new-in-portal-forarcgis-10-3.htm

Forero, E. y C. Romero. 2005. Estudios en leguminosas colombianas. Academia Colombiana de Ciencias Exactas, Físicas y Naturales. Bogotá, D.C., Colombia. 413 pp.

Galvis, G. y L. Mesa. 2014. Consideraciones históricas sobre la distribución actual del bosque seco en Colombia. In: Pizano, C. y H. García (eds.). El Bosque Seco Tropical en Colombia. Instituto de Investigación de Recursos Biológicos Alexander von Humboldt (IAvH). Bogotá, D.C., Colombia. Pp. 24-33

García, H., E. Carbonó-De la Hoz y W. Barranco-Pérez. 2021. Diversidad beta del bosque seco tropical en el norte del Caribe colombiano. Revista de la Academia Colombiana de Ciencias Exactas, Físicas y Naturales 45(174): 95-108. DOI: https://doi.org/10.18257/raccefyn.1267

García, M., A. Piñeros, F. Bernal y E. Ardila. 2012. Variabilidad climática, cambio climático y el recurso hídrico en Colombia. Revista de Ingeniería 36: 60-64. DOI: https://doi. org/10.16924/revinge.36.11

Gentry, A. 1982. Patterns of Neotropical Plant Species Diversity. In: Hecht, M., B. Wallace y G. Prance (eds.). Evolutionary Biology. Springer. Boston, MA., USA. Pp. 1-84. DOI: https:// doi.org/10.1007/978-1-4615-6968-8_1

Gentry, A. 1995. Diversity and floristic composition of neotropical dry forests. In: Medina, E., H. Mooney y S. Bullock (eds.). Seasonally Dry Tropical Forests. Cambridge University Press. Cambridge, UK. Pp. 146-194.

Gentry, A. 1996. A field guide to the families and genera of woody plants of northwest South America (Colombia, Ecuador, Peru), with supplementary notes on herbaceous taxa. The University of Chicago Press. Chicago, USA. 920 pp.
Gentry, A. 2009. Bignoniaceae. In: Forero, E., J. Betancur, G. Galeano y J. Aguirre (eds.). Flora de Colombia $n^{\circ} 25$. Universidad Nacional de Colombia, Facultad de Ciencias, Instituto de Ciencias Naturales. Bogotá, Colombia. 462 pp.

Herazo, F., J. Mercado y H. Mendoza. 2017. Estructura y Composición Florística del Bosque Seco Tropical en los Montes de María (Sucre-Colombia). Ciencia en Desarrollo 8(1): 71-82. DOI: https://doi.org/10.19053/01217488.v8.n1.2017.5912

Herrera, Y., L. Pérez y V. Londoño. 2017. Flora. In: Vásquez, A. (ed.). Guía de especies de flora y fauna de la Ciénaga de La Virgen, sector UCG6. Jardín Botánico de Cartagena “Guillermo Piñeres". Cartagena, Colombia. Pp. 33-129.

Holdridge, L. 1967. Life Zone Ecology. Tropical Science Center. San José, Costa Rica. 206 pp.

IAVH. 1998. El bosque seco tropical (Bs-T) en Colombia. Instituto de Investigación de Recursos Biológicos Alexander von Humboldt (IAVH). Programa de Inventario de la Biodiversidad Grupo de Exploraciones y Monitoreo Ambiental GEMA. Bogotá, D.C., Colombia. 24 pp.

IAVH. 2014. Bosques secos tropicales de Colombia, escala 1:100.000. Instituto de Investigación de Recursos Biológicos Alexander von Humboldt (IAVH). Bogotá, D.C., Colombia.

IDEAM. 2020. Instituto de Hidrología, Meterología y Estudios Ambientales. Consulta y descarga de datos hidrometeorológicos. http://dhime.ideam.gov.co/atencionciudadano/ (consultado enero de 2020).

IDEAM, IGAC, IAVH, INVEMAR, SINCHI y IIAP. 2007. Ecosistemas continentales, costeros y marinos de Colombia. Instituto de Hidrología, Meteorología y Estudios Ambientales (IDEAM), Instituto Geográfico Agustín Codazzi (IGAC), Instituto Alexander von Humboldt (IAVH), Instituto de Investigaciones Marinas y Costeras José Benito Vives De Andréis (INVEMAR), Instituto Amazónico de Investigaciones Científicas (SINCHI), Instituto de Investigaciones Ambientales del Pacífico (IIAP). Imprenta Nacional de Colombia. Bogotá, D.C., Colombia. $276 \mathrm{pp}$.

IPNI. 2020. International Plant Names Index. The Royal Botanic Gardens, Kew, Harvard University Herbaria \& Libraries and Australian National Botanic Gardens. http://www.ipni.org (consultado abril de 2020).

Jiménez, A. y J. Hortal. 2003. Las curvas de acumulación de especies y la necesidad de evaluar la calidad de los inventarios biológicos. Revista Ibérica de Aracnología 8: 151-161. 
JSTOR. 2020. JSTOR Global Plants. http://www.jstor.org/ (cosultado abril de 2020).

Klein, T., D. Yakir, N. Buchmann y J. Grünzweig. 2014. Towards an advanced assessment of the hydrological vulnerability of forests to climate change-induced drought. New Phytologist 201(3): 712-716. DOI: https://doi.org/10.1111/nph.12548

Kohler, T., A. Wehrli y M. Jurek. 2014. Las montañas y el cambio climático una preocupación mundial. Serie sobre el Desarrollo Sostenible de las Regiones de Montaña. Centro para el Desarrollo y el Medio Ambiente (CDE), Agencia Suiza para el Desarrollo y la Cooperación (COSUDE) y Geographica Bernensia. Lima, Perú. 136 pp.

Londoño, V. y A. Torres. 2015. Estructura y composición vegetal de un bosque seco tropical en regeneración en Bataclán (Cali, Colombia). Colombia Forestal 18(1): 71-85. DOI: https:// doi.org/10.14483/udistrital.jour.colomb.for.2015.1.a04

Méndez, M., A. Castro, R. Barrantes, C. Pacheco y C. Ramírez. 2005. Eficacia de dos tipos de recolecta para registrar la diversidad de melolóntidos nocturnos (Coleoptera: Scarabaeoidea). Acta Zoológica Mexicana 21: 109-124.

Mendoza, H. 1999. Estructura y riqueza florística del bosque seco tropical en la región caribe y el valle del río Magdalena, Colombia. Caldasia 21(1): 70-94.

Mendoza, H., B. Ramírez y L. Jiménez. 2004. Rubiaceae de Colombia. Ramos López Editorial. Bogotá, D.C., Colombia. 351 pp.

Morales, F. 2009. Estudios en las Apocynaceae neotropicales XXXIX: revisión de las Apocynoideae y Rauvolfioideae de Honduras. Anales del Jardín Botánico de Madrid 66(2): 217262. DOI: https://doi.org/10.3989/ajbm.2205

Morillas, L., J. Durán, A. Rodríguez, J. Roales, A. Gallardo, G. Lovett y P. Groffman. 2015. Nitrogen supply modulates the effect of changes in drying-rewetting frequency on soil C and $\mathrm{N}$ cycling and greenhouse gas exchange. Global Change Biology 21(10): 3854-3863. DOI: https://doi.org/10.1111/ gcb. 12956

Noguera, A. 2016. Cambio climático y degradación del bosque seco. In: Noguera, A. (ed.). Bosque seco y cambio climático. Universidad Nacional Agraria (UNA). Managua, Nicaragua. Pp. 1-8.

Pabón, J. y J. Montealegre. 2000. La variabilidad climática interanual asociada al ciclo El Niño-La Niña-Oscilación del Sur y su efecto en el patrón pluviométrico de Colombia. Meteorología Colombiana 2: 7-21.
Pinto, J. y J. Rangel. 2010. Flora asociada a las formaciones paramunas de la cordillera Occidental y el norte de Colombia (Sierra Nevada de Santa Marta, Serranía de Perijá), con base en parcelas de campo. In: Rangel-Churio, J. O. (ed.). Colombia diversidad biótica $\mathrm{X}$ : Cambio global (natural) y climático (antrópico) en el páramo colombiano. Universidad Nacional de Colombia. Bogotá, D.C., Colombia. Pp. 411-442.

Pizano, C., R. González, M. F. González, F. Castro, R. López, N. Rodríguez, A. Idárraga, W. Vargas, H. Vergara y A. Castaño. 2014. Las plantas de los bosques secos de Colombia. In: Pizano, C. y H. García (eds.). El Bosque Seco Tropical en Colombia. Instituto de Investigación de Recursos Biológicos Alexander von Humboldt (IAvH). Bogotá, D.C., Colombia. Pp. 48-93.

Rangel, O. 2012. Colombia Diversidad Biótica XII. La Región Caribe de Colombia. Editorial CÓDICE Ltda. Bogotá, D.C., Colombia. 1046 pp.

Rangel, O. y A. Garzón. 1995. Sierra Nevada de Santa Marta (Con énfasis en la parte norte Transecto del Rio Buritaca-La Cumbre). In: Rangel, O. Colombia, Diversidad Biótica II. Instituto de Ciencias Naturales. Bogotá, D.C., Colombia. Pp. 155-170.

Reich, P. y R. Borchert. 1982. Phenology and ecophysiology of the tropical tree, Tabebuia neochrysantha (Bignoniaceae). Ecology 63(2): 294-299. DOI: https://doi.org/10.2307/1938945

Ruiz, M. y G. Bernal. 2009. Variabilidad estacional e interanual del viento en los datos del reanálisis NCEP/NCAR en la cuenca Colombia, mar Caribe. Avances en Recursos Hidráulicos 20: 7-20.

Smith, L. y A. Fernández. 1954. Revision violacearum colombiae. Caldasia 6(28): 83-181.

Statsoft. 2001. STATISTICA. Version 6, (data analysis software system and computer program manual). StatSoft, Inc. Tulsa, USA. https://statistica.software.informer.com/6.0/

Torres, A., J. Adarve, M. Cárdenas, J. Vargas, V. Londoño, K. Rivera, J. Home, O. Duque y A. González. 2012. Dinámica sucesional de un fragmento de bosque seco tropical del Valle del Cauca, Colombia. Biota Colombiana 13: 66-85. DOI: https:// doi.org/10.15468/lbinut

TROPICOS. 2020. Tropicos.org. Missouri Botanical Garden. https://tropicos.org (consultado marzo de 2020).

UNAL. 2019. Universidad Nacional de Colombia. Colecciones en Línea. http://www.biovirtual.unal.edu.co (consultado marzo de 2020). 
USBG. 2020. United States Botanic Garden. Collection. http:// www.usbg.gov/search-collection (consultado abril de 2020).

Van Der Heijden, G. y O. Phillips. 2009. Environmental effects on Neotropical liana species richness. Journal of Biogeography 36(8): 1561-1572. DOI: https://doi.org/10.1111/j.13652699.2009.02099.x

Vargas, J., A. Gonzales, E. Barona y W. Bolivar. 2016. Vegetation Composition and Structure of Tropical Dry Forest Fragments and of Two Sites with Anthropic Activity in La Dorada and Victoria, Caldas. Revista de Ciencias 20: 13-60.

Vargas, W. 2012. Los bosques secos del Valle del Cauca, Colombia: una aproximación a su flora actual. Biota Colombiana 13: 102-164. DOI: http://doi.org/10.15472/9iusrz
Villanueva, B., O. Melo y M. Rincón. 2015. Estado del conocimiento y aportes a la flora vascular del bosque seco del Tolima. Colombia Forestal 18(1): 9-23. DOI: https://doi. org/10.14483/udistrital.jour.colomb.for.2015.1.a01

Villareal, H., M. Álvarez, S. Córdoba, F. Escobar, G. Fagua, F. Gast, H. Mendoza, M. Ospina y A. Umaña. 2006. Manual de métodos para el desarrollo de inventarios de biodiversidad. Programa de Inventarios de Biodiversidad. Instituto de Investigación de Recursos Biológicos Alexander von Humboldt. Bogotá, D.C., Colombia. 236 pp.

Wright, J. 2005. Tropical forests in a changing environment. Trends in Ecology and Evolution 20(10): 553-560. DOI: https://doi. org/10.1016/j.tree.2005.07.009 
Apéndice: Especies registradas en los diferentes sitios de muestreo: finca La Patagonia (A-LP), municipio Aracataca; finca Monato (A-M), municipio Aracataca; finca Villa Lorena (A-VL), municipio Aracataca; finca Italia, municipio Sabanas de San Ángel (S.S.A); finca Silvaderal, municipio Santa Bárbara de Pinto (S.B.P) y finca Tacaloa, municipio Plato (P).

\begin{tabular}{|c|c|c|c|c|c|c|c|}
\hline TAXÓN & HÁBITO & A-LP & A-M & $A-V L$ & S.S.A & S.B.P & $\mathrm{P}$ \\
\hline \multicolumn{8}{|l|}{ ANGIOSPERMAS } \\
\hline \multicolumn{8}{|l|}{ MAGNOLIIDES } \\
\hline \multicolumn{8}{|l|}{ ANNONACEAE } \\
\hline Annona reticulata $\mathrm{L}$. & árbol & $x$ & & & & & \\
\hline \multicolumn{8}{|l|}{ ARISTOLOCHIACEAE } \\
\hline Aristolochia maxima Jacq. & liana & & $x$ & $x$ & & & \\
\hline \multicolumn{8}{|l|}{ LAURACEAE } \\
\hline Nectandra turbacensis (Kunth) Nees & árbol & & & $x$ & & & \\
\hline Ocotea celastroides (Meisn.) Mez & árbol & & $x$ & & & & \\
\hline \multicolumn{8}{|l|}{ MONOCOTILEDÓNEAS } \\
\hline \multicolumn{8}{|l|}{ ARACEAE } \\
\hline Monstera adansonii Schott & liana & $x$ & & & & & \\
\hline Philodendron jacquinii Schott & liana & $x$ & & & & & \\
\hline \multicolumn{8}{|l|}{ ARECACEAE } \\
\hline Attalea butyracea (Mutis ex L.f.) Wess. Boer & palma & & & $x$ & $x$ & & \\
\hline Bactris gasipaes Kunth & palma & $x$ & & & & & \\
\hline Bactris major Jacq. & palma & $x$ & & & & & \\
\hline Desmoncus orthacanthos Mart. & palma & & & $x$ & & & \\
\hline \multicolumn{8}{|l|}{ DIOSCOREACEAE } \\
\hline Dioscorea sp. & liana & & & & & $x$ & \\
\hline \multicolumn{8}{|l|}{ SMILACACEAE } \\
\hline Smilax spinosa Mill. & liana & $x$ & & $x$ & $x$ & & \\
\hline \multicolumn{8}{|l|}{ EUDICOTILEDÓNEAS } \\
\hline \multicolumn{8}{|l|}{ ACANTHACEAE } \\
\hline Aphelandra phlogea Leonard & arbusto & & & & & & $\mathrm{x}$ \\
\hline Aphelandra pulcherrima (Jacq.) Kunth & arbusto & & & $x$ & & & \\
\hline \multicolumn{8}{|l|}{ ACHATOCARPACEAE } \\
\hline Achatocarpus sp. & árbol & & & $x$ & & & \\
\hline \multicolumn{8}{|l|}{ ANACARDIACEAE } \\
\hline Anacardium excelsum (Kunth) Skeels & árbol & $\mathrm{x}$ & & & & & \\
\hline Astronium graveolens Jacq. & árbol & $x$ & $x$ & $x$ & $x$ & $x$ & $\mathrm{x}$ \\
\hline Spondias mombin L. & árbol & $x$ & $\mathrm{x}$ & $\mathrm{x}$ & $\mathrm{x}$ & & \\
\hline \multicolumn{8}{|l|}{ APOCYNACEAE } \\
\hline Aspidosperma polyneuron Müll. Arg. & árbol & & & $x$ & $x$ & $x$ & $x$ \\
\hline Forsteronia spicata (Jacq.) G. Mey. & liana & $\mathrm{x}$ & & & & & \\
\hline Marsdenia altissima (Jacq.) Dugand & liana & & & & & $x$ & \\
\hline Marsdenia macrophylla (Schult.) E. Fourn. & liana & $x$ & $x$ & & & & \\
\hline Mesechites sp. & liana & & & & & $x$ & $x$ \\
\hline Plumeria sp. & árbol & & & & & $x$ & \\
\hline Rauvolfia viridis Roem. \& Schult. & arbusto & & $x$ & & & & \\
\hline Stemmadenia grandiflora (Jacq.) Miers & árbol & $x$ & & & & & \\
\hline Tabernaemontana cymosa Jacq. & árbol & & & $x$ & & & \\
\hline
\end{tabular}


Apéndice: Continuación.

\begin{tabular}{|c|c|c|c|c|c|c|c|}
\hline TAXÓN & HÁBITO & A-LP & $A-M$ & $A-V L$ & S.S.A & S.B.P & $\mathrm{P}$ \\
\hline \multicolumn{8}{|l|}{ ARALIACEAE } \\
\hline Dendropanax arboreus (L.) Decne. \& Planch. & árbol & $\mathrm{x}$ & & & & & \\
\hline \multicolumn{8}{|l|}{ BIGNONIACEAE } \\
\hline Adenocalymma inundatum DC. & liana & $\mathrm{x}$ & $x$ & $x$ & & $\mathrm{x}$ & $\mathrm{x}$ \\
\hline Amphilophium paniculatum (L.) Kunth & liana & & & & & $x$ & \\
\hline Bignonia diversifolia Kunth & liana & $\mathrm{x}$ & $x$ & $x$ & $x$ & $\mathrm{x}$ & $\mathrm{x}$ \\
\hline Bignonia pterocalyx (Urb.) L.G. Lohmann & liana & & & & & $\mathrm{X}$ & $\mathrm{x}$ \\
\hline Crescentia cujete L. & árbol & & $x$ & & $x$ & & \\
\hline Dolichandra uncata (Andrews) L.G. Lohmann & liana & $x$ & $x$ & & & & \\
\hline Fridericia mollissima (Kunth) L.G. Lohmann & liana & & & $\mathrm{x}$ & & & \\
\hline Fridericia pubescens (L.) L.G. Lohmann & liana & & & & $x$ & $x$ & $\mathrm{x}$ \\
\hline Handroanthus billbergii (Bureau \& K. Schum.) S.O. Grose & árbol & & & & & $x$ & $x$ \\
\hline Handroanthus chrysanthus (Jacq.) S.O. Grose & árbol & & $\mathrm{x}$ & $\mathrm{x}$ & $\mathrm{x}$ & $\mathrm{x}$ & $\mathrm{x}$ \\
\hline Handroanthus ochraceus (Cham.) Mattos & árbol & & & & & $\mathrm{x}$ & \\
\hline Martinella obovata (Kunth) Bureau \& K. Schum. & liana & & & & & $x$ & \\
\hline Pleonotoma variabilis (Jacq.) Miers & liana & & & & & $x$ & $\mathrm{x}$ \\
\hline Roseodendron chryseum (S.F. Blake) Miranda & árbol & & & & $x$ & & \\
\hline Tanaecium jaroba Sw. & árbol & $x$ & & & & & \\
\hline Tanaecium pyramidatum (Rich.) L.G. Lohmann & liana & & & & $\mathrm{x}$ & & \\
\hline \multicolumn{8}{|l|}{ BIXACEAE } \\
\hline Cochlospermum vitifolium (Willd.) Spreng. & árbol & & $x$ & $x$ & & & \\
\hline \multicolumn{8}{|l|}{ BORAGINACEAE } \\
\hline Cordia alliodora (Ruiz \& Pav.) Oken & árbol & & $x$ & $x$ & $x$ & & $\mathrm{x}$ \\
\hline Cordia thaisiana G. Agostini & árbol & $x$ & $x$ & $x$ & $x$ & $x$ & $x$ \\
\hline Cordia sp. 1 & árbol & & & & $x$ & $x$ & \\
\hline Cordia sp. 2 & arbusto & & & & & & $x$ \\
\hline \multicolumn{8}{|l|}{ BURSERACEAE } \\
\hline Bursera simaruba (L.) Sarg. & árbol & $x$ & $x$ & & & & $x$ \\
\hline \multicolumn{8}{|l|}{ CACTACEAE } \\
\hline Hylocereus sp. & arbusto & & & & & $x$ & $x$ \\
\hline Opuntia caracassana Salm-Dyck & arbusto & & & & & $x$ & \\
\hline \multicolumn{8}{|l|}{ CAPPARACEAE } \\
\hline Belencita nemorosa (Jacq.) Dugand & árbol & & & & & $x$ & $x$ \\
\hline Capparidastrum frondosum (Jacq.) Cornejo \& Iltis & árbol & $x$ & & $x$ & $x$ & $x$ & \\
\hline Capparidastrum pachaca (Kunth) Hutch. & árbol & & & & & & $x$ \\
\hline Cynophalla flexuosa (L.) J. Presl & arbusto & & & & & $x$ & \\
\hline Cynophalla verrucosa & arbusto & & & & & $x$ & \\
\hline Quadrella indica (L.) Iltis \& Cornejo & árbol & & & & $x$ & $x$ & $x$ \\
\hline Quadrella odoratissima (Jacq.) Hutch. & árbol & & & & & $x$ & $x$ \\
\hline \multicolumn{8}{|l|}{ CELASTRACEAE } \\
\hline Hippocratea volubilis $\mathrm{L}$. & liana & & & & & $x$ & $x$ \\
\hline \multicolumn{8}{|l|}{ CHRYSOBALANACEAE } \\
\hline Licania cuspidata (Rusby) Prance & árbol & & & & & & $x$ \\
\hline Parinari pachyphylla Rusby & árbol & $x$ & $x$ & & & & \\
\hline
\end{tabular}


Apéndice: Continuación.

\begin{tabular}{|c|c|c|c|c|c|c|c|}
\hline TAXÓN & HÁBITO & A-LP & $A-M$ & $A-V L$ & S.S.A & S.B.P & $\mathrm{P}$ \\
\hline \multicolumn{8}{|l|}{ CLUSIACEAE } \\
\hline Garcinia intermedia (Pittier) Hammel & árbol & $\mathrm{x}$ & & & & & \\
\hline \multicolumn{8}{|l|}{ COMBRETACEAE } \\
\hline Combretum fruticosum (Loefl.) Stuntz & liana & & $x$ & $\mathrm{x}$ & & & \\
\hline Terminalia catappa $\mathrm{L}$. & árbol & & & & & & $x$ \\
\hline \multicolumn{8}{|l|}{ CONNARACEAE } \\
\hline Rourea glabra Kunth & liana & $x$ & & $x$ & & & \\
\hline \multicolumn{8}{|l|}{ DILLENIACEAE } \\
\hline Tetracera volubilis L. & liana & $\mathrm{x}$ & $x$ & $x$ & & & \\
\hline \multicolumn{8}{|l|}{ ELAEOCARPACEAE } \\
\hline Sloanea pubiflora Benth. & árbol & & $x$ & & & & \\
\hline Sloanea terniflora (DC.) Standl. & árbol & & & $x$ & & & \\
\hline \multicolumn{8}{|l|}{ EUPHORBIACEAE } \\
\hline Acalypha diversifolia Jacq. & arbusto & & & & & & $x$ \\
\hline Alchornea grandiflora Müll. Arg. & árbol & & & & $x$ & $x$ & \\
\hline Cnidoscolus longipes (Pax) I.M. Johnst. & arbusto & & & & & $\mathrm{x}$ & \\
\hline Cnidoscolus urens (L.) Arthur & arbusto & & & & & $x$ & \\
\hline Croton hondensis (H. Karst.) G.L. Webster & arbusto & & & $x$ & & & \\
\hline Croton malambo H. Karst. & árbol & & & & & & $\mathrm{x}$ \\
\hline Dalechampia scandens L. & liana & & $x$ & & & & \\
\hline Hura crepitans L. & árbol & & & $x$ & & & $x$ \\
\hline \multicolumn{8}{|l|}{ FABACEAE } \\
\hline Albizia carbonaria (Britton) Niezgoda \& Nevling & árbol & & & & & & $x$ \\
\hline Albizia niopoides (Benth.) Burkart & árbol & & & & $x$ & & \\
\hline Albizia pistaciifolia (Willd.) Barneby \& J.W. Grimes & árbol & & $\mathrm{x}$ & & & & \\
\hline Andira inermis (Wright) DC. & árbol & & $x$ & & & & \\
\hline Caesalpinia ebano H.Karst. & árbol & & & & $x$ & $x$ & $x$ \\
\hline Chloroleucon mangense (Jacq.) Britton \& Rose & árbol & & $x$ & & $x$ & & \\
\hline Entada polystachya (L.) DC. & liana & & & & $x$ & & \\
\hline Enterolobium cyclocarpum (Jacq.) Griseb. & árbol & & $x$ & $x$ & & & \\
\hline Erythrina velutina Willd. & árbol & & & & & & $x$ \\
\hline Hymenaea courbaril L. & árbol & & $x$ & & & & \\
\hline Inga sapindoides Willd. & árbol & $x$ & & $x$ & & & \\
\hline Inga tayronaensis T.D. Penn. & arbusto & $x$ & & & & & \\
\hline Libidibia coriaria (Jacq.) Schltdl. & árbol & & $x$ & & & $x$ & $\mathrm{x}$ \\
\hline Lonchocarpus velutinus Benth. & árbol & & & & $x$ & $x$ & $x$ \\
\hline Lonchocarpus sp. & árbol & & & $x$ & & & \\
\hline Machaerium arboreum (Jacq.) Vogel & árbol & & & $x$ & & & \\
\hline Machaerium biovulatum Micheli & árbol & & & & $x$ & $x$ & \\
\hline Machaerium bondaense Pittier & árbol & & & & $x$ & $x$ & $x$ \\
\hline Machaerium capote Dugand & árbol & $x$ & & $x$ & & $x$ & $x$ \\
\hline Machaerium glabratum Pittier & árbol & $x$ & $x$ & $x$ & & & \\
\hline Machaerium microphyllum (E. Mey.) Standl. & árbol & & $x$ & $x$ & & & \\
\hline Machaerium milleflorum Pittier & árbol & & & $x$ & & & \\
\hline
\end{tabular}


Apéndice: Continuación.

\begin{tabular}{|c|c|c|c|c|c|c|c|}
\hline TAXÓN & HÁBITO & A-LP & A-M & $A-V L$ & S.S.A & S.B.P & $\mathrm{P}$ \\
\hline Machaerium seemannii Seem. & árbol & & & $x$ & & $x$ & \\
\hline Machaerium sp. & árbol & & & & & & $x$ \\
\hline Mucuna mutisiana (Kunth) DC. & liana & & & $x$ & & & \\
\hline Mucuna pruriens (L.) DC. & liana & & $x$ & & & & \\
\hline Muellera burkartii M.Sousa & árbol & & & & $x$ & & \\
\hline Myrospermum frutescens Jacq. & árbol & $x$ & $x$ & $x$ & $\mathrm{x}$ & $\mathrm{x}$ & $\mathrm{x}$ \\
\hline Myroxylon balsamum (L.) Harms & árbol & & & $x$ & $x$ & $x$ & $x$ \\
\hline Ormosia colombiana Rudd & árbol & & & & & & $x$ \\
\hline Ormosia sp. 1 & árbol & & & & & & $x$ \\
\hline Ormosia sp. 2 & árbol & & & & & $\mathrm{x}$ & $\mathrm{x}$ \\
\hline Piscidia carthagenensis Jacq. & árbol & & & & & & $x$ \\
\hline Pithecellobium hymenaeifolium (Willd.) Benth. & árbol & & & & $\mathrm{X}$ & & \\
\hline Pithecellobium lanceolatum (Willd.) Benth. & árbol & & & & & $\mathrm{x}$ & $\mathrm{x}$ \\
\hline Platymiscium pinnatum (Jacq.) Dugand & árbol & $\mathrm{X}$ & $x$ & $x$ & $\mathrm{X}$ & & \\
\hline Platypodium elegans Vogel & árbol & & $x$ & $x$ & & & \\
\hline Pseudosamanea guachapele (Kunth) Harms & árbol & & $x$ & & & & \\
\hline Pterocarpus acapulcensis Rose & árbol & & $x$ & $\mathrm{x}$ & & & \\
\hline Pterocarpus officinalis Jacq. & árbol & & & & & $\mathrm{x}$ & \\
\hline Schnella glabra (Jacq.) Dugand & liana & $\mathrm{X}$ & $x$ & $\mathrm{x}$ & & $\mathrm{X}$ & $\mathrm{x}$ \\
\hline Senegalia polyphylla (DC.) Britton & árbol & & & & & $\mathrm{x}$ & \\
\hline Senegalia riparia (Kunth) Britton & arbusto & $\mathrm{x}$ & & & $x$ & & \\
\hline Senegalia tamarindifolia (L.) Britton \& Rose & árbol & & & & $\mathrm{X}$ & $\mathrm{X}$ & $x$ \\
\hline Senna atomaria (L.) H.S.Irwin \& Barneby & árbol & & & & $\mathrm{x}$ & & \\
\hline Vachellia macracantha (Willd.) Seigler \& Ebinger & árbol & & & & $\mathrm{x}$ & & \\
\hline Zygia inaequalis (Willd.) Pittier & árbol & & & & & & $x$ \\
\hline \multicolumn{8}{|l|}{ LAMIACEAE } \\
\hline Vitex compressa Turcz. & árbol & & $x$ & $x$ & & $x$ & $\mathrm{x}$ \\
\hline \multicolumn{8}{|l|}{ LECYTHIDACEAE } \\
\hline Lecythis minor Jacq. & árbol & & $\mathrm{x}$ & & & & \\
\hline \multicolumn{8}{|l|}{ MALPHIGIACEAE } \\
\hline Amorimia concinna (C.V. Morton) W.R. Anderson & liana & $\mathrm{x}$ & & $x$ & & & \\
\hline Diplopterys lucida (Rich.) W.R. Anderson \& C. Davis & liana & & & & & $\mathrm{x}$ & $\mathrm{x}$ \\
\hline Heteropterys sp. 1 & liana & & & & $\mathrm{x}$ & & \\
\hline Heteropterys sp. 2 & arbusto & & & $x$ & & & \\
\hline Malpighia glabra L. & árbol & & & & $x$ & $\mathrm{x}$ & $\mathrm{x}$ \\
\hline Mascagnia sp. 1 & liana & & & & $x$ & & $x$ \\
\hline \multicolumn{8}{|l|}{ MALVACEAE } \\
\hline Apeiba tibourbou Aubl. & árbol & $x$ & & $x$ & & & \\
\hline $\begin{array}{l}\text { Bastardiopsis myrianthus (Triana \& Planch.) Fuertes \& } \\
\text { Fryxell }\end{array}$ & árbol & & & $x$ & & & \\
\hline Byttneria aculeata Jacq. & liana & & & & & $x$ & $\mathrm{x}$ \\
\hline Ceiba pentandra (L.) Gaertn. & árbol & $x$ & & & & & \\
\hline Guazuma ulmifolia Lam. & árbol & $x$ & $x$ & $x$ & $x$ & $x$ & \\
\hline Luehea seemannii Triana \& Planch. & árbol & $x$ & & $x$ & & & \\
\hline
\end{tabular}


Apéndice: Continuación.

\begin{tabular}{|c|c|c|c|c|c|c|c|}
\hline TAXÓN & HÁBITO & A-LP & A-M & $A-V L$ & S.S.A & S.B.P & $\mathrm{P}$ \\
\hline Pseudobombax septenatum (Jacq.) Dugand & árbol & $\mathrm{x}$ & $x$ & $x$ & $x$ & $x$ & $x$ \\
\hline Sterculia apetala (Jacq.) H. Karst. & árbol & $x$ & & $\mathrm{x}$ & $\mathrm{x}$ & & \\
\hline \multicolumn{8}{|l|}{ MELIACEAE } \\
\hline Guarea guidonia (L.) Sleumer & árbol & $x$ & & & & & \\
\hline Trichilia acuminata (Schult.) C. DC. & árbol & & & & & $\mathrm{x}$ & $x$ \\
\hline Trichilia hirta L. & árbol & & $\mathrm{x}$ & & & & \\
\hline \multicolumn{8}{|l|}{ MORACEAE } \\
\hline Brosimum alicastrum Sw. & árbol & $x$ & & & & $x$ & \\
\hline Ficus insipida Willd. & árbol & $x$ & & & & & \\
\hline Sorocea sprucei (Baill.) J.F. Macbr. & árbol & $\mathrm{x}$ & & $x$ & & & $x$ \\
\hline \multicolumn{8}{|l|}{ MUNTINGIACEAE } \\
\hline Muntingia calabura L. & árbol & & $x$ & & & & \\
\hline \multicolumn{8}{|l|}{ MYRTACEAE } \\
\hline Eugenia procera (Sw.) Poir. & arbusto & & $x$ & & & & \\
\hline Eugenia uniflora L. & arbusto & & & & & $x$ & $x$ \\
\hline Myrcia fallax (Rich.) DC. & árbol & & & & & $x$ & \\
\hline \multicolumn{8}{|l|}{ NYCTAGINACEAE } \\
\hline Guapira uberrima (Standl.) Lundell & árbol & & & & & $\mathrm{x}$ & \\
\hline \multicolumn{8}{|l|}{ OPILIACEAE } \\
\hline Agonandra brasiliensis Benth. & árbol & & & & & & $x$ \\
\hline \multicolumn{8}{|l|}{ PHYTOLACCACEAE } \\
\hline Seguieria aculeata Jacq. & liana & & & & $x$ & $x$ & $x$ \\
\hline Seguieria americana L. & arbusto & & & & & $x$ & \\
\hline \multicolumn{8}{|l|}{ POLYGONACEAE } \\
\hline Coccoloba padiformis Meisn. & árbol & & & & $\mathrm{x}$ & & \\
\hline Ruprechtia ramiflora (Jacq.) C.A. Mey. & árbol & & & & & $\mathrm{x}$ & $x$ \\
\hline Triplaris americana $\mathrm{L}$. & árbol & $x$ & $\mathrm{x}$ & $x$ & $x$ & & \\
\hline \multicolumn{8}{|l|}{ PRIMULACEAE } \\
\hline Clavija sanctae-martae B.Ståhl & árbol & $x$ & $x$ & $x$ & & & \\
\hline Stylogyne turbacensis (Kunth) Mez & árbol & $x$ & & & & & \\
\hline \multicolumn{8}{|l|}{ RHAMNACEAE } \\
\hline Ziziphus sp. & arbusto & & & & $x$ & & $x$ \\
\hline \multicolumn{8}{|l|}{ RUBIACEAE } \\
\hline Alseis mutisii Moldenke & arbusto & & & & & & $x$ \\
\hline Calycophyllum candidissimum (Vahl) DC. & árbol & $x$ & & $x$ & & & \\
\hline Chomelia spinosa Jacq. & arbusto & & & & & $x$ & \\
\hline Coussarea grandifolia Rusby & árbol & & & $x$ & & & \\
\hline Coutarea hexandra (Jacq.) K. Schum. & árbol & & $x$ & & & & \\
\hline Faramea occidentalis (L.) A. Rich. & árbol & $x$ & & & & & \\
\hline Genipa americana L. & árbol & & $\mathrm{x}$ & $x$ & $x$ & & \\
\hline Randia aculeata $\mathrm{L}$. & arbusto & & & & $x$ & & \\
\hline Randia armata (Sw.) DC. & arbusto & $x$ & $x$ & & $x$ & $x$ & \\
\hline Randia dioica $\mathrm{H}$. Karst. & arbusto & & & & $x$ & & \\
\hline Randia sp. & arbusto & & & & $\mathrm{X}$ & $x$ & \\
\hline
\end{tabular}


Apéndice: Continuación.

\begin{tabular}{|c|c|c|c|c|c|c|c|}
\hline TAXÓN & HÁBITO & A-LP & A-M & $A-V L$ & S.S.A & S.B.P & $\mathrm{P}$ \\
\hline Rosenbergiodendron formosum (Jacq.) Fagerl. & árbol & & & & & & $x$ \\
\hline Simira cordifolia (Hook. f.) Steyerm. & árbol & $x$ & & & & & \\
\hline \multicolumn{8}{|l|}{ RUTACEAE } \\
\hline Zanthoxylum caribaeum Lam. & árbol & & & & $x$ & & \\
\hline \multicolumn{8}{|l|}{ SALICACEAE } \\
\hline Casearia corymbosa Kunth & árbol & $\mathrm{x}$ & $\mathrm{x}$ & $x$ & $x$ & & \\
\hline Casearia sylvestris Sw. & árbol & & & & & $x$ & \\
\hline Casearia zizyphoides Kunth & árbol & & & & & $x$ & $x$ \\
\hline Casearia sp. 1 & árbol & & & & & & $x$ \\
\hline Casearia sp. 2 & árbol & & & & $x$ & $x$ & $x$ \\
\hline Xylosma flexuosa (Kunth) Hemsl. & arbusto & $x$ & $x$ & $x$ & & & \\
\hline \multicolumn{8}{|l|}{ SAPINDACEAE } \\
\hline Cupania latifolia Kunth & árbol & & & & $x$ & & \\
\hline Melicoccus oliviformis Kunth & árbol & $\mathrm{x}$ & $\mathrm{x}$ & $\mathrm{x}$ & & $\mathrm{x}$ & $x$ \\
\hline Paullinia pinnata $\mathrm{L}$. & liana & & & & & $x$ & $x$ \\
\hline Sapindus saponaria L. & árbol & & & & $\mathrm{x}$ & & $x$ \\
\hline \multicolumn{8}{|l|}{ SAPOTACEAE } \\
\hline Pradosia colombiana (Standl.) T.J. Ayers \& Boufford & árbol & & & & $\mathrm{x}$ & & \\
\hline \multicolumn{8}{|l|}{ SIMAROUBACEAE } \\
\hline Quassia amara $\mathrm{L}$. & árbol & $x$ & & $x$ & & & \\
\hline \multicolumn{8}{|l|}{ ULMACEAE } \\
\hline Ampelocera edentula Kuhlm. & árbol & $x$ & & & & & $x$ \\
\hline \multicolumn{8}{|l|}{ URTICACEAE } \\
\hline Cecropia peltata L. & árbol & & & $x$ & & & \\
\hline \multicolumn{8}{|l|}{ VIOLACEAE } \\
\hline Hybanthus sp. & arbusto & & & & $\mathrm{x}$ & & \\
\hline \multicolumn{8}{|l|}{ VITACEAE } \\
\hline Vitis tiliifolia Schult. & liana & & $\mathrm{x}$ & & & & \\
\hline \multicolumn{8}{|l|}{ ZYGOPHYLLACEAE } \\
\hline Bulnesia arborea (Jacq.) Engl. & árbol & & & & $\mathrm{x}$ & $x$ & $x$ \\
\hline TOTAL & & 55 & 51 & 61 & 56 & 68 & 67 \\
\hline
\end{tabular}

